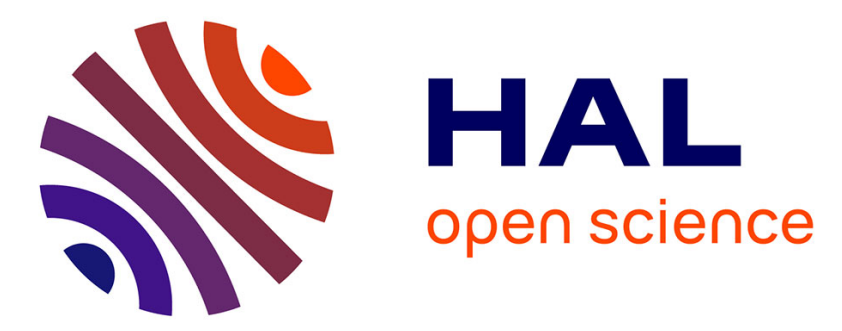

\title{
A new robust D-stability condition for real convex polytopic uncertainty
}

Dimitri Peaucelle, Denis Arzelier, Olivier Bachelier, Jacques Bernussou

\section{To cite this version:}

Dimitri Peaucelle, Denis Arzelier, Olivier Bachelier, Jacques Bernussou. A new robust D-stability condition for real convex polytopic uncertainty. Systems and Control Letters, 2000, 40 (1), pp.21-30. 10.1016/S0167-6911(99)00119-X . hal-03310541

\section{HAL Id: hal-03310541 \\ https://hal.laas.fr/hal-03310541}

Submitted on 30 Jul 2021

HAL is a multi-disciplinary open access archive for the deposit and dissemination of scientific research documents, whether they are published or not. The documents may come from teaching and research institutions in France or abroad, or from public or private research centers.
L'archive ouverte pluridisciplinaire HAL, est destinée au dépôt et à la diffusion de documents scientifiques de niveau recherche, publiés ou non, émanant des établissements d'enseignement et de recherche français ou étrangers, des laboratoires publics ou privés. 


\title{
A new robust $\mathcal{D}$-stability condition for real convex polytopic uncertainty
}

\author{
D. Peaucelle - D. Arzelier - O. Bachelier - J. Bernussou \\ $L A A S$ - CNRS \\ 7, Avenue du Colonel Roche \\ 31077 Toulouse cedex - France \\ Tel. 0561336200 \\ fax. 0561336969 \\ email: peaucell@laas.fr - arzelier@laas.fr - bachelie@laas.fr - bernusou@laas.fr \\ Paper scl 99-070
}

\begin{abstract}
The problem of robust $\mathcal{D}$-stability analysis with respect to real convex polytopic uncertainties is tackled. A new $\mathcal{L} \mathcal{M} I$-based sufficient condition for the existence of parameter-dependent Lyapunov functions is proposed. This condition generalises previously published conditions. Numerical comparisons with quadratic stability results as well as previous results based on parameterdependent Lyapunov functions illustrate the relevance of this new condition. Finally, this result appears to be promising for robust multi-objective performance analysis and control synthesis purposes.
\end{abstract}

Keywords: Robust stability, quadratic stability, parametric uncertainty, $\mathcal{L} \mathcal{M} I$, parameter-dependent Lyapunov functions.

\section{Introduction}

Even though the problem of assessing robustness of stability and performance against real parametric uncertainty is known to be in general $\mathcal{N} \mathcal{P}$-hard, numerous more or less conservative results are disseminated in the related literature. Among all these results, four main streams may be quoted. The algebraic approaches based on Kharitonov's theorem, [3], generally propose nice robust stability tests which suffer from restrictive assumptions and are hardly generalisable to robust synthesis problems. In the $\mu / k_{m}$ theory, [23], a very general and well-established framework including real parametric as well as neglected dynamics is available. Nevertheless, it leads to heavy numerical procedures for which the well-kwown frequency sweep is still necessary. Strongly related to this last approach, the methods based on topological separation have proven their efficiency. Particularly, in [13], an exact characterisation of $\mu$ is given whatever the properties of the uncertainty are. For numerical purpose, it is then necessary to use appropriate relaxations of the derived condition.

Very appealing methods have been developped in the context of Lyapunov theory. In particular, many results are based on the concept of quadratic stability, [5], [6], [10], [18]. Unfortunately, these approaches may be very conservative due to the use of a single Lyapunov function for the entire uncertainty set. The reduction of conservatism necessarily implies the use of parameter-dependent Lyapunov functions, [7], [9], [16]. While in [7], [9], robust stability conditions involving parameterdependent Lyapunov functions are developped for continuous-time uncertain systems, in [16], a new 
robust stability condition is derived for discrete-time uncertain systems.

The contribution of this paper consists of the derivation of a new robust $\mathcal{D}_{R}$-stability condition, (i.e. robust stability with respect to some $\mathcal{D}_{R}$ subregion of the complex plane), with respect to real convex polytopic uncertainty, generalising the one in [16] and leading to identical result for the special case presented in [7]. The interest of this new condition mainly relies on the additional degrees of freedom supplied by the introduction of new matrix variables. Beyond the advances in the reduction of conservativeness for robust stability analysis, the new condition appears to be very promising for robust multi-objective performance analysis as well as for robust synthesis purposes. We would like to stress that in the particular case of robust stability analysis, similar ideas emerged in the other independent works, [11], [17].

The paper is organised as follows. In the next section, the definitions and Lyapunov characterisations of $\mathcal{D}_{R}$-stability and quadratic $\mathcal{D}_{R}$-stability are presented and different classes of subregions of the complex plane are recalled. In the third section, the new robust $\mathcal{D}_{R}$-stability condition based on parameter-dependent Lyapunov functions is derived. The relevance of this condition is illustrated by different numerical experiments in section 4. Finally, the conclusion section deals with the promising perspectives offered by this new result.

Notation is standard. The transpose of a matrix $A$ is denoted $A^{\prime}$ and $A^{*}$ reads for complex conjugate transpose. For symmetric matrices, $>(\geq)$ denotes the Löewner partial order, i.e. $A>(\geq) B$ iff $A-B$ is positive (semi) definite. $\delta$ is the derivation operator for continuous-time systems, $(\delta[x(t)]=\dot{x}(t))$ and the delay operator for discrete-time ones, $(\delta[x(t)]=x(t+1)) . \otimes$ is the Kronecker product of two matrices. We remind that $(A \otimes B)(C \otimes D)=(A C) \otimes(C D)$.

\section{The robust $\mathcal{D}_{R}$-stability analysis problem}

\subsection{A new characterisation of $\mathcal{L} \mathcal{M} I$ regions}

Let a dynamical system be given by its state equation $\delta[x(t)]=A x(t)$ and let $\mathcal{D}$ be a subregion of the complex plane $\mathbb{C}$. The problem of finding easy testable conditions for the matrix $A$ to have all its roots in the subregion $\mathcal{D}$, (the problem of deciding if $A$ is $\mathcal{D}$-stable), has been thoroughly investigated in the last years. In [12], the notion of polynomial regions has been introduced.

$$
\mathcal{D}_{P}=\left\{z \in \mathbb{C}: \sum_{0 \leq k, l \leq m} c_{k l} z^{k} z^{* l}<0\right\}
$$

An extended Lyapunov theorem is then proposed for such regions in [14] and [12]. In [6], an $\mathcal{L} \mathcal{M} I$ based characterisation of matrix root-clustering regions for a given matrix $A$ is presented. The main result consists in the derivation of an extended Lyapunov matrix equation for a new class of convex subregions of the complex plane named the $\mathcal{L} \mathcal{M} I$ regions and characterised by an $\mathcal{L} \mathcal{M} I$ in $z$ and $z^{*}$.

$$
\mathcal{D}_{L}=\left\{z \in \mathbb{C}: R_{11}+R_{12} z+R_{12}^{\prime} z^{*}<\mathbf{0}\right\}
$$

where $R_{11} \in \mathbb{R}^{d \times d}$ is a symmetric matrix and $R_{12} \in \mathbf{R}^{d \times d}$, [6]. In this paper, we focus our attention on $\mathcal{L} \mathcal{M} I$ regions. Indeed, due to the polynomial nature of their Lyapunov characterisation, problems such as robust analysis or synthesis are not easily tractable for polynomial regions. The characterisation of $\mathcal{L} \mathcal{M} I$ regions is now revisited. For such a purpose, we define a new class of subregions of the complex plane: 


\section{Definition 1}

Let $R \in \mathbb{R}^{2 d \times 2 d}$ be a symmetric matrix partitionned as:

$$
R=\left[\begin{array}{ll}
R_{11} & R_{12} \\
R_{12}^{\prime} & R_{22}
\end{array}\right]: \begin{aligned}
& R_{11}=R_{11}^{\prime} \in \mathbf{R}^{d \times d} \\
& R_{22}=R_{22}^{\prime} \in \mathbf{R}^{d \times d}
\end{aligned}
$$

We define the $\mathcal{D}_{R}$ region of the complex plane:

$$
\mathcal{D}_{R}=\left\{z \in \mathbb{C}: R_{11}+R_{12} z+R_{12}^{\prime} z^{*}+R_{22} z z^{*}<0\right\}
$$

Without any assumption on the matrix $R_{22}, \mathcal{D}_{R}$ regions are not convex, but with the assumption of positive definiteness, $R_{22} \geq \mathbf{0}$, (4) appears to be a slight modification of the characterisation of $\mathcal{L} \mathcal{M} I$ regions.

\section{Remarks 1}

- As for $\mathcal{L} \mathcal{M} I$ regions the $\mathcal{D}_{R}$ regions are symmetric with respect to the real axis.

- When $R_{22} \geq \mathbf{0}$, it can be rewritten as $R_{22}=L L^{\prime}$ where $L \in \mathbf{R}^{d \times d}$ is any square root of $R_{22}$. In this case, the $\mathcal{D}_{R}$ regions are just another formulation for $\mathcal{L} \mathcal{M} I$ regions:

$$
\left[\begin{array}{ll}
\mathbf{1}_{d} & \mathbf{1}_{d} z^{*}
\end{array}\right] R\left[\begin{array}{c}
\mathbf{1}_{d} \\
\mathbf{1}_{d} z
\end{array}\right]<\mathbf{0} \quad R_{22}=L L^{\prime} \Leftrightarrow\left[\begin{array}{cc}
R_{11}+R_{12} z+R_{12}^{\prime} z^{*} & L z \\
L^{\prime} z^{*} & -\mathbf{1}_{d}
\end{array}\right]<\mathbf{0}
$$

Note that the class of $\mathcal{L} \mathcal{M} I$ regions belongs to the class of $\mathcal{D}_{R}$ regions but our investigations will be restricted to the former, i.e. we assume that $R_{22} \geq \mathbf{0}$.

\section{Remarks 2}

The characterisation proposed in definition 1 is not only a formal result but will appear to be usefull to simplify the different expressions. This is easily seen when $\mathcal{D}_{R}$ is chosen to be a disk. Indeed, the particular choices for $R$ :

$$
R \in \mathbb{R}^{2 \times 2} \quad R_{c}=\left[\begin{array}{c|c}
0 & 1 \\
\hline 1 & 0
\end{array}\right], R_{d}=\left[\begin{array}{c|c}
-1 & 0 \\
\hline 0 & 1
\end{array}\right]
$$

correspond to the usual criteria for stability of respectively continuous-time and discrete-time systems. Note that the $\mathcal{L} \mathcal{M} I$ formulation of [6] for the unit disk is equivalent to choose $R$ as:

$$
R \in \mathbb{R}^{4 \times 4} \quad R_{d} \mathcal{L M} I=\left[\begin{array}{cc|cc}
-1 & 0 & 0 & 1 \\
0 & -1 & 0 & 0 \\
\hline 0 & 0 & 0 & 0 \\
1 & 0 & 0 & 0
\end{array}\right]
$$

For a more complete description of possible $\mathcal{L} \mathcal{M} I$-regions, the interested reader may have a look to the references [6], [2].

\section{Definition 2}

A matrix $A \in \mathbf{R}^{n \times n}$ is said to be $\mathcal{D}_{R}$-stable if and only if all its eigenvalues lie in the $\mathcal{D}_{R}$ region defined by (4).

An $\mathcal{L} \mathcal{M} I$ characterisation for $\mathcal{D}_{R}$-stability can be easily deduced from [6].

\section{Theorem 1}

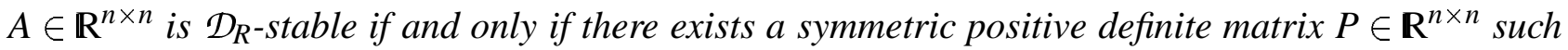
that:

$$
R_{11} \otimes P+R_{12} \otimes(P A)+R_{12}^{\prime} \otimes\left(A^{\prime} P\right)+R_{22} \otimes\left(A^{\prime} P A\right)<\mathbf{0}
$$

The proof is easily derived by following the proof for $\mathcal{L} \mathcal{M} I$ regions in [6]. 


\subsection{Robust and quadratic $\mathcal{D}_{R}$-stability}

We consider now an uncertain LTI dynamical system modelled as:

$$
\delta[x(t)]=A x(t) \quad A \in \mathcal{A}
$$

where $\mathcal{A}$ is a polytope of $\mathbf{R}^{n \times n}$ with $N$ vertices $A_{1}, \cdots, A_{N}$. $\mathcal{A}$ can be formally defined as:

$$
\mathcal{A}=\left\{A \in \mathbb{R}^{n \times n}: A=\sum_{i=1}^{N} \tau_{i} A_{i}, \tau_{i} \geq 0, \forall i \in\{1, \cdots, N\}, \sum_{i=1}^{N} \tau_{i}=1\right\}
$$

Note that $\mathcal{A}$ is a convex and bounded domain. This representation is quite general and emcompasses for instance the well-known case of interval matrices. Moreover, this type of uncertainty may be considered as structured uncertainty. The problem of analysing stability of all the realisations of $A \in \mathcal{A}$ is therefore $\mathcal{N} \mathcal{P}$-hard. For robustness analysis purpose, two different types of stability are usually encountered in the literature. While robust stability ensures that the uncertain system is stable for all admissible constant and unknown parameter realisations, quadratic stability guarantees stability via the existence of a quadratic Lyapunov function for all possibly time-varying parameter variations.

Definition 3 [23]

$\mathcal{A}$ is robustly $\mathcal{D}_{R}$-stable, if and only if, for all $A \in \mathcal{A}, A$ is $\mathcal{D}_{R}$-stable.

Thus $\mathcal{A}$ is robustly $\mathcal{D}_{R}$-stable if and only if, for each $A \in \mathcal{A}$, there exists a symmetric positive definite matrix $P$ such that (8) holds.

Definition 4 [10]

$\mathcal{A}$ is quadraticaly $\mathcal{D}_{R}$-stable, if and only if, there exists a symmetric positive definite matrix $P$ such that for all $A \in \mathcal{A}$ :

$$
R_{11} \otimes P+R_{12} \otimes(P A)+R_{12}^{\prime} \otimes\left(A^{\prime} P\right)+R_{22} \otimes\left(A^{\prime} P A\right)<\mathbf{0}
$$

Before exposing the main result and for comparison sake, we first recall the $\mathcal{L} \mathcal{M} I$-type necessary and sufficient condition for quadratic $\mathcal{D}_{R}$-stability of $\mathcal{A}$.

\section{Theorem 2}

$\mathcal{A}$ is quadratically $\mathcal{D}_{R}$-stable, if and only if, there exists a unique symmetric definite positive matrix $P \in \mathbb{R}^{n \times n}$ such that for all $i \in\{1, \cdots, N\}$ :

$$
\mathcal{L}(P)=\left[\begin{array}{cc}
R_{11} \otimes P+R_{12} \otimes\left(P A_{i}\right)+R_{12}^{\prime} \otimes\left(A_{i}^{\prime} P\right) & \left(\mathbf{1}_{d} \otimes A_{i}^{\prime}\right)(L \otimes P) \\
\left(L^{\prime} \otimes P\right)\left(\mathbf{1}_{d} \otimes A_{i}\right) & -\mathbf{1}_{d} \otimes P
\end{array}\right]<\mathbf{0}
$$

where $R_{22}=L L^{\prime}$.

\section{$\underline{\text { Proof }}$}

Necessity is straightforward since if quadratic stability is satisfied for all the polytope it must be the case at all the vertices. Sufficiency is deduced from:

$$
\left[\begin{array}{cc}
R_{11} \otimes P+R_{12} \otimes(P A)+R_{12}^{\prime} \otimes\left(A^{\prime} P\right) & \left(\mathbf{1}_{d} \otimes A^{\prime}\right)(L \otimes P) \\
\left(L^{\prime} \otimes P\right)\left(\mathbf{1}_{d} \otimes A\right) & -\mathbf{1}_{d} \otimes P
\end{array}\right]=\sum_{i=1}^{N} \tau_{i} \mathcal{L}(P)<\mathbf{0}
$$


Applying a Schur complement argument leads to the quadratic $\mathcal{D}_{R}$-stability of (9).

Even if it has proved its efficiency for the development of synthesis procedures for various types of robust control problem, [5], [8], [18], quadratic stability is undoubtedly a stronger requirement than robust stability and is at the best an easily tractable sufficient condition for robust stability. The conservativeness of the approaches based on quadratic stability comes from the fact that $\mathcal{D}_{R}$-stability is proved through the use of a single quadratic Lyapunov function $v(x)=x^{\prime} P x$ for the whole uncertainty domain $\mathcal{A}$ while robust $\mathcal{D}_{R}$ stability may be proved by using a parameter-dependent Lyapunov function $v(x, \tau)=x^{\prime} P(\tau) x$. The next section focuses on this particular point and provides a new sufficient condition for robust $\mathcal{D}_{R}$-stability whose conservativeness appears to be similar or less important than that of previous existing conditions.

\section{A new robust $\mathcal{D}_{R}$-stability condition}

\subsection{A preliminary result}

Before stating the main result concerning the robust $\mathcal{D}_{R}$-stability problem, an instrumental theorem is given providing the key idea for the sequel.

\section{Theorem 3}

$A \in \mathbb{R}^{n \times n}$ is $\mathcal{D}_{R}$-stable if and only if there exists a symmetric positive definite matrix $P \in \mathbb{R}^{n \times n}$ and two matrices $F \in \mathbb{R}^{d n \times d n}, G \in \mathbb{R}^{d n \times d n}$ such that:

$$
\left[\begin{array}{cc}
R_{11} \otimes P+F\left(\mathbf{1}_{d} \otimes A\right)+\left(\mathbf{1}_{d} \otimes A^{\prime}\right) F^{\prime} & R_{12} \otimes P+\left(\mathbf{1}_{d} \otimes A^{\prime}\right) G-F \\
R_{12}^{\prime} \otimes P+G^{\prime}\left(\mathbf{1}_{d} \otimes A\right)-F^{\prime} & R_{22} \otimes P-G-G^{\prime}
\end{array}\right]<\mathbf{0}
$$

Proof of sufficiency.

Since the matrix $\left[\begin{array}{ll}\mathbf{1}_{d n} & \mathbf{1}_{d} \otimes A^{\prime}\end{array}\right]$ has full rank, (14) implies that:

$$
\left[\begin{array}{c}
\mathbf{1}_{d n} \\
\mathbf{1}_{d} \otimes A
\end{array}\right]^{\prime}\left[\begin{array}{cc}
R_{11} \otimes P+F\left(\mathbf{1}_{d} \otimes A\right)+\left(\mathbf{1}_{d} \otimes A^{\prime}\right) F^{\prime} & R_{12} \otimes P+\left(\mathbf{1}_{d} \otimes A^{\prime}\right) G-F \\
R_{12}^{\prime} \otimes P+G^{\prime}\left(\mathbf{1}_{d} \otimes A\right)-F^{\prime} & R_{22} \otimes P-G-G^{\prime}
\end{array}\right]\left[\begin{array}{c}
\mathbf{1}_{d n} \\
\mathbf{1}_{d} \otimes A
\end{array}\right]<\mathbf{0}
$$

which is exactly the expression (8).

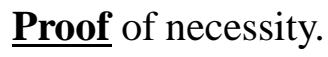

Suppose that there exists a symmetric positive definite matrix $P$ such that (8) holds. Necessarily, there exists a scalar $\alpha>0$ such that:

$$
R_{11} \otimes P+R_{12} \otimes(P A)+R_{12}^{\prime} \otimes\left(A^{\prime} P\right)+R_{22} \otimes\left(A^{\prime} P A\right)<-\alpha \mathbf{1}_{d} \otimes\left(A^{\prime} A\right)
$$

This may be rewriten also as:

$$
R_{11} \otimes P+R_{12} \otimes(P A)+R_{12}^{\prime} \otimes\left(A^{\prime} P\right)+\left(\mathbf{1}_{d} \otimes A^{\prime}\right)\left(R_{22} \otimes P+\alpha \mathbf{1}_{d n}\right)\left(\mathbf{1}_{d} \otimes A\right)<\mathbf{0}
$$

As $R_{22} \geq \mathbf{0}, P>0$ and $\alpha>0$ and by using a Schur complement argument this implies that:

$$
\left[\begin{array}{cc}
R_{11} \otimes P+R_{12} \otimes(P A)+R_{12}^{\prime} \otimes\left(A^{\prime} P\right) & \left(\mathbf{1}_{d} \otimes A^{\prime}\right)\left(R_{22} \otimes P+\alpha \mathbf{1}_{d n}\right) \\
\left(R_{22} \otimes P+\alpha \mathbf{1}_{d n}\right)\left(\mathbf{1}_{d} \otimes A\right) & -\left(R_{22} \otimes P+\alpha \mathbf{1}_{d n}\right)
\end{array}\right]<\mathbf{0}
$$


Taking $G=R_{22} \otimes P+\frac{\alpha}{2} \mathbf{1}_{d n}$ and $F=R_{12} \otimes P-\frac{\alpha}{2}\left(\mathbf{1}_{d} \otimes A^{\prime}\right)$ it means that there exist $P=P^{\prime}>\mathbf{0}$ and matrices $G \in \mathbf{R}^{d n \times d n}$ and $F \in \mathbf{R}^{d n \times d n}$ such that:

$$
\left[\begin{array}{cc}
R_{11} \otimes P+F\left(\mathbf{1}_{d} \otimes A\right)+\left(\mathbf{1}_{d} \otimes A^{\prime}\right) F^{\prime}+\alpha\left(\mathbf{1}_{d} \otimes\left(A^{\prime} A\right)\right) & R_{12} \otimes P+\left(\mathbf{1}_{d} \otimes A^{\prime}\right) G-F \\
R_{12}^{\prime} \otimes P+G^{\prime}\left(\mathbf{1}_{d} \otimes A\right)-F^{\prime} & R_{22} \otimes P-G-G^{\prime}
\end{array}\right]<\mathbf{0}
$$

\section{Remarks 3}

For continuous-time and discrete-time systems, the condition (14) respectively reads:

$$
\left[\begin{array}{cc}
\left(A^{\prime} F^{\prime}+F A\right) & P-F+A^{\prime} G \\
P-F^{\prime}+G^{\prime} A & -\left(G+G^{\prime}\right)
\end{array}\right]<\mathbf{0}\left[\begin{array}{cc}
-P+\left(A^{\prime} F^{\prime}+F A\right) & -F+A^{\prime} G \\
-F+G^{\prime} A & P-\left(G+G^{\prime}\right)
\end{array}\right]<\mathbf{0}
$$

In [11], [17], similar results are independently proposed for diagonal and simultaneous stability of polytope of matrices using the passivity and real-positiveness approach. In [16], by choosing $F=\mathbf{0}$ in the discrete-time condition, a particular discrete-time robust stability condition is given for control synthesis purpose.

\subsection{The main result}

The main result is now stated.

\section{Theorem 4}

If, there exists $F \in \mathbb{R}^{d n \times d n}, G \in \mathbb{R}^{d n \times n}$ and $N$ symmetric positive definite matrices $P_{i}$ such that:

$$
\forall i \in\{1, \cdots, N\}\left[\begin{array}{cc}
R_{11} \otimes P_{i}+F\left(\mathbf{1}_{d} \otimes A_{i}\right)+\left(\mathbf{1}_{d} \otimes A_{i}^{\prime}\right) F^{\prime} & R_{12} \otimes P_{i}+\left(\mathbf{1}_{d} \otimes A_{i}^{\prime}\right) G-F \\
R_{12}^{\prime} \otimes P_{i}+G^{\prime}\left(\mathbf{1}_{d} \otimes A_{i}\right)-F^{\prime} & R_{22} \otimes P_{i}-G-G^{\prime}
\end{array}\right]<\mathbf{0}
$$

then (9) is robustly $\mathcal{D}_{R}$-stable.

\section{$\underline{\text { Proof }}$}

The proof is similar to the proof for quadratic stability of theorem 2 with a parameter-dependent Lyapunov matrix:

$$
P(\tau)=\sum_{i=1}^{N} \tau_{i} P_{i}
$$

\section{Remarks 4}

- (12) is a sufficient condition for (21). The proposed condition of robust stability therefore includes quadratic stability.

- In the particularly interesting cases of robust stability for continuous-time or discrete-time uncertain systems, (21) respectively reads, $\forall i \in\{1, \cdots, N\}$ :

$$
\left[\begin{array}{cc}
\left(A_{i}^{\prime} F^{\prime}+F A_{i}\right) & P_{i}-F+A_{i}^{\prime} G \\
P_{i}-F^{\prime}+G^{\prime} A_{i} & -\left(G+G^{\prime}\right)
\end{array}\right]<\mathbf{0}\left[\begin{array}{cc}
-P_{i}+\left(A_{i}^{\prime} F^{\prime}+F A_{i}\right) & -F+A_{i}^{\prime} G \\
-F+G^{\prime} A_{i} & P_{i}-\left(G+G^{\prime}\right)
\end{array}\right]<\mathbf{0}
$$


Keeping in mind the reference [16], it is interesting to know if condition (21) may be used for $\mathcal{D}_{R}$ stabilisability problems. Let us consider the following uncertain system:

$$
\delta[x(t)]=A x(t)+B u(t) \quad\left[\begin{array}{ll}
A & B
\end{array}\right] \in \mathcal{A B}
$$

where $u \in \mathbf{R}^{q}$ are the inputs and $\mathcal{A B}$ is a polytope of $\mathbf{R}^{n \times(n+q)}$ with $N$ vertices $\left[A_{1} B_{1}\right], \cdots,\left[A_{N} B_{N}\right]$. We look for a condition of state-feedback $\mathcal{D}_{R}$-stabilisability, that is, for a condition such that $A+B K$ is robustly $\mathcal{D}_{R}$-stable when $K$ is a state-feedback gain of $\mathbb{R}^{q \times n}$. The following theorem is a hint for further work on robust control. We assume for this result that $R_{22}>\mathbf{0}$, the $\mathcal{D}_{R}$ region is compact.

\section{Theorem 5}

If there exists $H \in \mathbf{R}^{n \times n}, S \in \mathbb{R}^{q \times n}$ and $N$ symmetric positive definite matrices $X_{i}$ such that $\forall i \in$ $\{i, \cdots, N\}$ :

$$
\left[\begin{array}{cc}
R_{11} \otimes X_{i}+R_{12} \otimes\left(A_{i} H+B_{i} S\right)+R_{12}^{\prime} \otimes\left(A_{i} H+B_{i} S\right)^{\prime} & R_{12}^{\prime} \otimes\left(X_{i}-H^{\prime}\right)+R_{22} \otimes\left(A_{i} H+B_{i} S\right) \\
R_{12} \otimes\left(X_{i}-H\right)+R_{22} \otimes\left(A_{i} H+B_{i} S\right)^{\prime} & R_{22} \otimes\left(X_{i}-H-H^{\prime}\right)
\end{array}\right]<\mathbf{0}
$$

then (24) is robustly $\mathcal{D}_{R}$-stabilisable by state-feedback and an admissible gain is:

$$
K=S H^{-1}
$$

\section{$\underline{\text { Proof }}$}

$\widehat{R_{22}}$ and the matrices $X_{i}$ are positive definite, therefore $H+H^{\prime}>\mathbf{0}, H$ is non singular. Take $K=S H^{-1}$, $A_{i}^{c l .}=A_{i}+B_{i} K, F=R_{12}^{\prime} \otimes H^{\prime}$ and $G=R_{22} \otimes H$, the condition writes then as:

$$
\left[\begin{array}{cc}
R_{11} \otimes X_{i}+F\left(\mathbf{1} \otimes A_{i}^{c l}\right)^{\prime}+\left(\mathbf{1} \otimes A_{i}^{c l .}\right) F^{\prime} & R_{12}^{\prime} \otimes X_{i}+\left(\mathbf{1} \otimes A_{i}^{c l .}\right) G-F \\
R_{12} \otimes X_{i}+G^{\prime}\left(\mathbf{1} \otimes A_{i}^{c l .}\right)^{\prime}-F^{\prime} & R_{22} \otimes X_{i}-G-G^{\prime}
\end{array}\right]<\mathbf{0}
$$

which is a transposed version of theorem 4.

\section{Remarks 5}

- For the particular case of discrete-time uncertain systems, the robust stabilisability by statefeedback is the same as in [16].

- For continuous-time systems, the above result is not applicable since $R_{22}=0$. Other methods may be proposed, (see [1]).

- The condition of theorem 5 is a sufficient condition for quadratic $\mathcal{D}_{R}$-stabilisability by statefeedback.

- The control gain does not depend on the Lyapunov function, which is new compared to the quadratic stability framework. This fact is quite valuable for further synthesis results. 


\section{Numerical experiments}

\subsection{Experiments 1}

Two different numerical experiments have been performed about robust stability of respectively continuous-time systems, (table 1) and discrete-time systems, (table 2).

We aim at comparing the sufficient condition (21) with previous results from [7] and [9]. Note that due to the restrictive nature of the uncertainty considered in [7], the uncertain matrix $A$ evolves in a hyperrectangle defined as follows,

$$
\dot{x}(t)=A_{0}+\sum_{i=1}^{L} A_{i} \delta_{i} \quad\left|\delta_{i}\right| \leq 1, i=1, \cdots, L
$$

For such uncertain models, the sufficient condition (21) may be simplified. It is therefore possible to seek a parameter-dependent Lyapunov function parameterized as,

$$
P(\delta)=P_{0}+\sum_{i=1}^{L} \delta_{i} P_{i}
$$

A new sufficient condition is then easily deduced.

\section{Theorem 6}

If, there exists $F \in \mathbb{R}^{n \times n}, G \in \mathbf{R}^{n \times n}$ and $L+1$ symmetric matrices $P_{i}, i=0,1, \cdots, L$ such that:

$$
\forall i \in\{1, \cdots, N\}\left[\begin{array}{cc}
A_{0}^{\prime} F^{\prime}+F A_{0}+F A \Delta_{i}^{\prime}+\Delta_{i} A^{\prime} F^{\prime} & P_{0}-F+P \Delta_{i}^{\prime}+\Delta_{i} A^{\prime} G \\
P_{0}-F^{\prime}+P \Delta_{i}+G^{\prime} A \Delta_{i}^{\prime} & -G-G^{\prime}
\end{array}\right]<\mathbf{0}
$$

where $P=\left[\begin{array}{lll}P_{1} & \cdots & P_{L}\end{array}\right]$ and $\Delta^{\prime}=\left[\begin{array}{lll}\delta_{1} \mathbf{1}_{n} & \cdots & \delta_{L} \mathbf{1}_{n}\end{array}\right]$.

Note that condition (30) may be easily extended to deal with robust $\mathcal{D}_{R}$-stability. then (9) is robustly $\mathcal{D}_{R}$-stable.

Five different methods are then compared on randomly generated continuous-time systems where $n$ is the size of the state vector while $L=2$ is the number of uncertain parameters. $A_{0}$ was chosen randomly and stable. The different tests are the quadratic stability test (12), test $c_{1}$, the sufficient condition from [9], test $c_{2}$, the sufficient condition from [7], test $c_{3}$, the sufficient condition (30), test $c_{4}$, the sufficient condition (21), test $c_{5}$. In the following table, the percentages of success of the analysis methods are given for about 5000 randomly generated continuous-time systems. These tests have been performed by using MATLAB and the $\mathcal{L} \mathcal{M} I$ solver SdPSOL, [22].

\begin{tabular}{|c|c|c|c|c|c|}
\hline $\mathrm{n}$ & test $c_{1}$ & test $c_{2}$ & test $c_{3}$ & test $c_{4}$ & test $c_{5}$ \\
\hline 2 & $52.2 \%$ & $60.3 \%$ & $69.8 \%$ & $71.1 \%$ & $71.3 \%$ \\
\hline 3 & $51.7 \%$ & $59.5 \%$ & $65.4 \%$ & $70.4 \%$ & $70.4 \%$ \\
\hline 4 & $36.4 \%$ & $47 \%$ & $50.9 \%$ & $57.1 \%$ & $57.4 \%$ \\
\hline 5 & $34.2 \%$ & $50.6 \%$ & $51 \%$ & $58.6 \%$ & $59.3 \%$ \\
\hline
\end{tabular}

The tests $c_{4}$ and $c_{5}$ prove to be less conservative than the previous existing methods. The test $c_{5}$ appears to be the more demanding computationally when compared to tests $c_{2}, c_{3}$ and $c_{4}$ when applied to uncertain systems of the form (28). In this particular case, the number of the $\mathcal{L} \mathcal{M} I$ constraints as 
well as the number of the variables grows exponentially with the number of uncertain parameters for test $c_{5}$ while the number of the $\mathcal{L} \mathcal{M} I$ constraints grows exponentially for tests $c_{4}$ and $c_{2}$. It has been observed that the reduction of the conservatism proposed by tests $c_{4}$ and $c_{5}$ grows with the number of vertices and with the size $n$ of the state vector.

For discrete-time uncertain systems, three conditions are compared, quadratic stability test, test $d_{1}$, the condition proposed in [16], test $d_{2}$, and the condition (21), test $d_{3}$. The polytopes of systems are randomly generated as for continuous-time stability analysis where $N$ is the number of vertices. Each vertex of the polytopes has at least one eigenvalue whose modulus is equal to 0.9 and other eigenvalues have a modulus less than 0.9 .

\begin{tabular}{|c|c|c|c|c|}
\hline $\mathrm{n}$ & $\mathrm{N}$ & test $d_{1}$ & test $d_{2}$ & test $d_{3}$ \\
\hline \multirow{3}{*}{4} & 3 & $8.8 \%$ & $68.6 \%$ & $91.0 \%$ \\
& 4 & $1.0 \%$ & $44.0 \%$ & $75.4 \%$ \\
& 5 & $0.2 \%$ & $27.4 \%$ & $63.0 \%$ \\
\hline \multirow{3}{*}{5} & 3 & $7.6 \%$ & $68.6 \%$ & $91.4 \%$ \\
& 4 & $0.8 \%$ & $46.4 \%$ & $82.8 \%$ \\
& 5 & $0.2 \%$ & $28.2 \%$ & $71.2 \%$ \\
\hline
\end{tabular}

The results obtained on many randomly generated polytopes of matrices clearly show the reduction of conservativeness introduced by the condition (21) with respect to existing tests. The test $d_{3}$ involves more variables than the tests $d_{1}$ and $d_{2}$. Precisely, the test $d_{3}$ involves, for $d=1,2 n^{2}+N \frac{n(n+1)}{2}$ additional variables as compared to the quadratic stability test and $n^{2}$ additional variables as compared to the test $d_{2}$. Nevertheless, when performing timing numerical experiments, the reduction of the conservatism far outweights the increased complexity. These timing experiments have been performed by using the $\mathcal{L} \mathcal{M} I$ solver of MATLAB on a SUN SPARC station ultra 5.

\begin{tabular}{|c|c|c|c|c|}
\hline $\mathrm{n}$ & $\mathrm{N}$ & test $d_{1}$ & test $d_{2}$ & test $d_{3}$ \\
\hline \multirow{3}{*}{10} & 3 & $0.64 \mathrm{~s}$ & $2.8 \mathrm{~s}$ & $4.7 \mathrm{~s}$ \\
& 4 & $1.2 \mathrm{~s}$ & $6.9 \mathrm{~s}$ & $10.6 \mathrm{~s}$ \\
& 5 & $3.1 \mathrm{~s}$ & $15.7 \mathrm{~s}$ & $25.7 \mathrm{~s}$ \\
\hline \multirow{3}{*}{5} & 3 & $0.1 \mathrm{~s}$ & $0.3 \mathrm{~s}$ & $0.5 \mathrm{~s}$ \\
& 4 & $0.2 \mathrm{~s}$ & $0.7 \mathrm{~s}$ & $1.2 \mathrm{~s}$ \\
& 5 & $0.4 \mathrm{~s}$ & $1.5 \mathrm{~s}$ & $2.5 \mathrm{~s}$ \\
\hline
\end{tabular}

\subsection{Experiments 2}

Up to this point we compared the method of theorem 4 to stability analysis methods in the case of continuous-time or discrete-time systems for which exist some earlier results. The goal of the following simple academic example is to illustrate the relevance of the method for more involved $\mathcal{L} \mathcal{M} I$ regions such as the intersection of a disk and a half-plane. The following regions are defined:

i) $\operatorname{Re}(z)<-0.9$ :

$$
R=\left[\begin{array}{c|c}
1.8 & 1 \\
\hline 1 & 0
\end{array}\right]
$$

ii) $\operatorname{Re}(z)<-0.9$ and $\|z\|<2.1$ :

$$
R=\left[\begin{array}{cc|cc}
1.8 & 0 & 1 & 0 \\
0 & -4.41 & 0 & 0 \\
\hline 1 & 0 & 0 & 0 \\
0 & 0 & 0 & 1
\end{array}\right]
$$




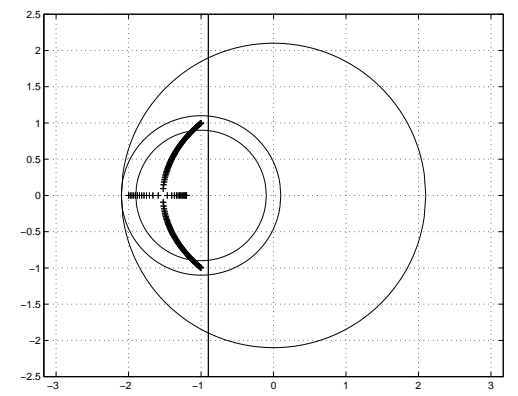

Figure 1: $\mathcal{D}_{R}$ regions and pole location

iii) $\operatorname{Re}(z)<-0.9$ and $\|z+1\|<1.1 \quad$ :

$$
R=\left[\begin{array}{cc|cc}
1.8 & 0 & 1 & 0 \\
0 & -0.21 & 0 & 1 \\
\hline 1 & 0 & 0 & 0 \\
0 & 1 & 0 & 1
\end{array}\right]
$$

iv) $\operatorname{Re}(z)<-0.9$ and $\|z+1\|<0.9$ :

$$
R=\left[\begin{array}{cc|cc}
1.8 & 0 & 1 & 0 \\
0 & 0.19 & 0 & 1 \\
\hline 1 & 0 & 0 & 0 \\
0 & 1 & 0 & 1
\end{array}\right]
$$

Let $\mathcal{A}$ be the polytope of matrices defined by two vertices:

$$
B_{1}=\left[\begin{array}{cc}
-1 & 1 \\
-1 & -1
\end{array}\right] \quad B_{2}=\left[\begin{array}{cc}
-2 & 0 \\
-1 & -1.2
\end{array}\right]
$$

The figure 1 shows, for the polytope $\mathcal{A}$ the variation of the eigenvalues when the uncertain parameter is varying from 0 to 1 . Quadratic $\mathcal{D}_{R}$-stability analysis succeeds only on region $i$ ) while the new $\mathcal{D}_{R}$-stability analysis of theorem 4 succeeds on all three first cases and of course fails on $i v$ ).

\section{Conclusion}

A new $\mathcal{L} \mathcal{M} I$-based robust $\mathcal{D}_{R}$-stability condition with respect to real convex polytopic uncertainties has been proposed. This test involves parameter-dependent Lyapunov functions which are linear with respect to the parametric uncertainty. It encompasses tests based on quadratic stability as well as a new recent result proposed in [16] and is similar to the condition proposed in [11], [17], for the particular case of robust stability. When dealing with the type of structured uncertainty presented in [7], the results are similar or even better.

Moreover, this condition is very promising for robust performance analysis and synthesis problems. Indeed, the extension of this result for the analysis of robust $\mathcal{D}_{R}$ stability and robust performance with respect to real convex polytopic uncertainties as well as dissipative linear fractional transformation, $\mathcal{L} \mathcal{F} \mathcal{T}$, uncertainty via parameter-dependent Lyapunov functions is quite straightforward, [19]. One additional interesting aspect of the given result is that it can be used to cope with robust multi-objective stabilisability problems. Using parameter-dependent Lyapunov functions and this new stability condition leads to construct robust control laws not directly depending upon the Lyapunov matrices, [1], unlike the methods developped in the quadratic stability framework and using the Lyapunov Shaping Paradigm, [21]. 


\section{Acknowledgement}

The authors are grateful to the referees for their valuable suggestions and many constructive comments which improved this paper, especially concerning the references [11], [17] to which our's attention was turned.

\section{References}

[1] D.Arzelier, D. Peaucelle, "Robust multi-objective output-feedback control for real parametric uncertainties via parameter-dependent Lyapunov functions", submitted to ROCOND 2000, september 1999.

[2] O. Bachelier, B. Pradin, "Bounds for uncertain matrix root-clustering in a union of subregions", International Journal of Robust and Non-linear Control, Vol. 9, No. 6, May 1999.

[3] B.R. Barmish, H.I. Kang, "A survey of extreme point results for robustness of control systems", Automatica, Vol. 29, pp. 13-35, 1993.

[4] S. Boyd, Q. Yang, "Structured and simultaneous Lyapunov functions for systems stability problems", Int. Journal of Control, Vol. 49, pp. 2215-2240, 1989.

[5] S. Boyd, L. El Ghaoui, E. Feron, V. Balakrishnan, Linear Matrix Inequalities in System and Control Theory, SIAM Studies in Applied Mathematics, 1994.

[6] M. Chilali, P. Gahinet, " $\mathcal{H}_{\infty}$ Design with pole placement constraints: an $\mathcal{L} \mathcal{M} I$ approach", IEEE Transactions on Automatic Control, Vol. AC-41, No. 3, pp. 358-367, 1996.

[7] E. Feron, P. Apkarian, P. Gahinet, "Analysis and synthesis of robust control systems via parameterdependent Lyapunov functions", IEEE Transactions on Automatic Control, Vol. AC-41, No. 7, pp. 1041-1046, 1996.

[8] P. Gahinet, A. Nemirovski, A.J. Laub, M. Chilali, " $\mathcal{L} \mathcal{M} I$ Control Toolbox User's Guide", The Mathworks Partner Series, 1995.

[9] P. Gahinet, P. Apkarian, M. Chilali, "Affine parameter-dependent Lyapunov functions and real parametric uncertainty", IEEE Transactions on Automatic Control, Vol. AC-41, No. 3, pp. 436-442, 1996.

[10] J.C. Geromel, P.L.D. Peres, J. Bernussou, "On a convex parameter space method for linear control design of uncertain systems", SIAM J. on Cont. Opt., Vol. 29, pp. 381-402, 1991.

[11] J.C. Geromel, M.C. de Oliveira, L. Hsu, "LMI Characterization of structural and robust stability", Linear Algebra and its Applications, vol. 285, 1-3, pp. 69-80, December 1998.

[12] S. Gutman, E.I. Jury, "A general theory for matrix root clustering in subregion of the complex plane", IEEE Transactions on Automatic Control, Vol. AC-26, pp. 853-863, 1981.

[13] T. Iwasaki, S. Hara, "Well-posedness of feedback systems: insights into exact robustness analysis and approximate computations", IEEE Transactions on Automatic Control, Vol. 43, No. 5, pp. 619630, 1998.

[14] A.G. Mazco, "The Lyapunov matrix equation for a certain class of regions bounded by algebraic curves", Soviet. Automatic Control, No. 13, pp. 37-42, 1980. 
[15] Y. Nesterov, A. Nemirovskii, Interior-Point Polynomial Algorithms in Convex Programming, SIAM Studies in Applied Mathematics, 1994.

[16] M.C. de Oliveira, J. Bernussou, J.C. Geromel, ”A new discrete-time robust stability condition", Systems \& Control Letters, Vol. 37, No. 4, July 1999.

[17] M.C. de Oliveira, J.C. Geromel, L. Hsu, "LMI Characterization of structural and robust stability: the discrete-time case", Linear Algebra and its Applications, Vol. 296, 1-3, pp.27-38, July 1999.

[18] D. Peaucelle, D. Arzelier, D. Henrion, "Performance and Quadratic Stabilisability via Dynamic Output Feedback for Uncertain Generalised Models", Proceedings of the 14th World IFAC Congress, Beijing, P.R. China, July 1999.

[19] D. Peaucelle, D. Arzelier, "Robust performance analysis $\mathcal{L} \mathcal{M} I$-based methods for real parametric uncertainty via parameter-dependent Lyapunov functions", submitted to IEEE Transactions on Automatic Control, June 1999.

[20] M.G. Safonov, R.Y. Chiang, "Real/complex $k_{m}$-synthesis without curve fitting”, in Control and dynamics systems, Vol. 56 (part 2), C.T. Leondes (Ed.), Academic Press, 1993.

[21] C. Scherer, P. Gahinet, M. Chilali, "Multiobjective output-feedback control via $\mathcal{L} \mathcal{M} I$ optimisation”, IEEE Transactions on Automatic Control, Vol. 42, No. 7, pp. 896-911, 1997.

[22] S.P. Wu, S. Boyd, "Design and implementation of a parser/solver for SDP with matrix structure", IEEE Conference on Computer Aided Control System Design, 1996.

[23] K.Zhou, J.C. Doyle, K. Glover, Robust and optimal control, Prentice Hall, New Jersey, 1996. 